\title{
QUAL $O$ PAPEL DO MÉDICO NA AVALIAÇÃO DE SEGURANÇA DE MEDICAMENTOS?
}

Muito se discute sobre a segurança de medicamentos, mas historicamente a classe médica encontra dificuldades em participar do processo necessário para garantir a segurança deste que é o armamentário diário da prática médica. Isto, obviamente, se deve a vários fatores e um deles é a discussão e entendimento do papel que o médico deve e precisa ocupar no sistema de identificação, avaliação, manejo e comunicação dos eventos adversos.

Eventos adversos são definidos como qualquer eventualidade médica num paciente recebendo um medicamento, que vão desde sinais e sintomas até condições sindrômicas. Fala-se de uma reação adversa quando existe a suspeita de uma relação do medicamento ao evento, e deve-se também ter em mente que quando uma reação não é descrita na informação ou bula do medicamento e dita não esperada, categoria que deve ser considerada de alta prioridade, principalmente se for considerada uma condição séria.

Um evento adverso é classificado como sério quando resulta em morte, ameaça à vida, leva à hospitalização ou torna-se debilitante. Defeitos congênitos também são eventos considerados sérios.

Fica claro que julgamento médico e científico têm de ser exercidos na decisão e uma reação adversa deve ser comunicada imediatamente ao fabricante do medicamento e autoridades sanitárias, de forma a permitir o início do processo que é chamado de farmacovigilância e que em última análise é a identificação, 
avaliação e inequívoco estabelecimento da relação do evento ao medicamento em questão, o que finalmente levará à inclusão deste nas bulas disponíveis a profissionais de saúde e pacientes.

Normalmente é difícil determinar se uma droga é a causa de um evento. 0 médico é normalmente a pessoa mais indicada para avaliar esta relação, pois está familiarizado com a história médica do paciente.

Após a identificação, a subsequente análise é feita cuidadosamente por médicos e cientistas especialistas em Segurança de Medicamentos de cada caso reportado. Nem sempre que um efeito é identificado e comunicado, significa que está definitivamente relacionada a uma droga. 0 problema pode ter se originado no uso de medicações concomitantes ou condições médicas preexistentes, aqui novamente o relato criterioso e detalhado do evento é de suma importância para a caracterização do mesmo.

A identificação de alguns de um dos seguintes fatores pode sugerir uma possível causa medicamentosa, quando por exemplo nenhuma outra medicação foi usada, ou quando ocorre uma melhora do evento imediatamente após a retirada da droga suspeita ou ainda quando há recorrência se a medicação é reintroduzida.

Atualmente, em todo o mundo, está se tornando prática comum a utilização de sistemas de Manejo de Riscos, um grupo de atividades de farmacovigilância desenhados para identificar, caracterizar, prevenir e minimizar riscos envolvendo produtos medicinais. Estes sistemas incluem também processos para avaliar a eficácia destas intervenções. Na Europa, Japão e EUA existem legislações que orientam a indústria farmacêutica quanto à necessidade, conteúdo e formato destes sistemas que são necessários para a aprovação da comercialização dos novos produtos. A Conferência Internacional para Harmonização $(\mathrm{ICH})$ de requisitos para o registro de produtos farmacêuticos para uso humano e um projeto que reúne as autoridades regulatórias da Europa, Japão e EUA e experts da indústria farmacêutica nas três regiões para discutir e criar guidelines baseadas nos aspectos técnicos e científicos para o registro de medicamentos é considerada em todo o mundo uma referência para estas atividades. Toda a indústria farmacêutica trabalha sob legislações baseadas nestes guidelines.

O manejo de riscos relacionados a um medicamento é um processo que ocorre desde o desenvolvimento em estudos pré-clínicos e clínicos e se estende durante toda a fase após a licença de comercialização. Normalmente, a licença é obtida baseada no balanço positivo do risco benefício do medicamento no uso em uma indicação para uma população específica. Isto significa que alguns problemas que podem não ter sido identificados na fase de desenvolvimento, seja devido ao número de pacientes que é restrito nos estudos clínicos, seja por uma restrição de uso durante aquele período em populações prédefinidas, que não inclui variações, como no caso da exclusão de populações de idosos e crianças nos estudos clínicos, no entanto estas populações poderão se beneficiar e fazer uso do medicamento na fase de comercialização.

0 processo de manejo de riscos consiste de quatro passos descritos na literatura como identificação de risco, avaliação do risco, minimização do risco e comunicação do risco e baseia se na premissa de que a combinação de múltiplos riscos que um medicamento oferece devem ser menores que os benefícios, respeitando uma margem de segurança maior possível, tanto para o indivíduo como para a população em geral.

Recentemente publicado (Janeiro de 2007) o volume 9A das Regras que Governam Produtos Medicinais na Europa (Guideline on Risk Management Systems for Medical Products for Human Use - Volume 9A) sob terceira revisão em 2009, estabelece que o Sistema de Manejamento de Risco deve conter duas partes: a primeira destinada à descrição do perfil de segurança do medicamento, particularmente no tempo e desenvolvimento, e um plano de farmacovigilância baseado no mesmo perfil de segurança.

A descrição das atividades quando da identificação de um risco passível de uma estratégia de minimização de risco deverá ser detalhada na segunda parte do plano, assim como uma proposta de avaliação de efetividade desta estratégia.

Este é um modelo que vem sendo adotado em todo o mundo e o Brasil como outros países vem implementando um modelo de farmacovigilância que visa garantir a segurança de pacientes.

O médico é peça fundamental deste sistema: é inegável que cada vez mais este papel deve ser exercido com responsabilidade e consciência, este papel é fundamental no aprofundamento do conhecimento dos medicamentos disponíveis, no desenvolvimento de estratégias para evitar seus eventos indesejados e ainda no desenvolvimento de novos medicamentos, contribuindo assim para que os pacientes recebam o melhor tratamento possível.

Aos médicos cabe assumir a criteriosa identificação, avaliação e relato às indústrias e autoridades competentes destes eventos, sem o qual se torna impossível iniciar o processo para que os mecanismos subsequentes de avaliação, confirmação da possível causalidade sejam estabelecidos e comunicados de forma efetiva aos interessados, profissionais de saúde e pacientes.

Senior Medical Consultant

Miriam Marotti

Prism Ideas (United Kingdon)

Regent House, Princes Court, Beam Heath Way, Nantwich, Cheshire, CW5 6PQ, England

miriam.marotti@prismideas.com

Referências

1. VOLUME 9A of "The Rules Governing Medicinal Products in the European Union Guidelines on Pharmacovigilance for Medicinal Products for Human Use". Sept.; 2008. Available from: http://ec.europa.eu/enterprise/pharmaceuticals/eudralex/vol-9/pdf/vol9a_09-2008.pdf.

2. International Conference on Harmonisation of Technical Requirements for Registration of Pharmaceuticals for Human use. Clinical Safety Data Management: definitions and standards for expedited reporting E2A (ICH E2A). Available from: http://www.ich.org/LOB/media/MEDIA436.pdf. 